\title{
'We spray and walk away': wall modifications decrease the impact of indoor residual spray campaigns through reductions in post-spray coverage
}

Mercy A. Opiyo ${ }^{1,2^{*}}$ and Krijn P. Paaijmans ${ }^{1,2,3,4}$

\begin{abstract}
Malaria prevalence has significantly reduced since 2000, largely due to the scale-up of vector control interventions, mainly indoor residual spraying (IRS) and long-lasting insecticide-treated nets (LLINs). Given their success, these tools remain the frontline interventions in the fight against malaria. Their effectiveness relies on three key ingredients: the intervention, the mosquito vector and the end-user. Regarding the intervention, factors such as the insecticide active ingredient(s) used and the durability and/or bio-efficacy of the tool over time are critical. For the vectors, these factors include biting and resting behaviours and the susceptibility to insecticides. Finally, the end-users need to accept and properly use the intervention. Whilst human attitude and behaviour towards LLINs are well-documented both during and after distribution, only initial coverage is monitored for IRS and in a few geographic settings the residual efficacy of the used product. Here, the historical evidence on end-users modifying their wall surfaces post-spraying is presented, a behaviour that has the potential to reduce actual IRS coverage, effectiveness and impact, as fewer people are truly protected. Therefore, clear guidelines on how to monitor IRS acceptability and/or coverage, both before, during and after spraying, are urgently needed as part of the Monitoring and Evaluation of malaria programmes.
\end{abstract}

Keywords: Insecticide, Wall modification, Residual efficacy, Communities, Vector control, Compliance, Elimination

\section{Background}

Insecticide-treated nets (ITNs), later replaced by longlasting insecticidal nets (LLINs), and indoor residual spraying (IRS) are core vector control interventions in malaria-endemic settings and have averted 663 million clinical malaria cases (three-quarters of the gains achieved) between 2000 and 2015 [1].

Albeit the huge success in the fight against malaria, malaria cases are on the rise again since $2016[2,3]$ which endangers the long-term goals of the Global Technical Strategy (GTS) for Malaria [4]. As LLINs and IRS will

\footnotetext{
*Correspondence: mercy.opiyo@isglobal.org

1 ISGlobal, Hospital Clinic, University of Barcelona, Barcelona, Spain

Full list of author information is available at the end of the article
}

remain the backbone of malaria control and elimination efforts, optimized approaches to improve these interventions are required to further reduce the burden.

In addition, current vector control tools face several challenges, which include sub-optimal user compliance [5] the rapid spread of insecticide resistance [6, 7] mosquito behavioural changes after implementation of interventions [8], and implementation costs. All these factors combined greatly undermine the effectiveness of current intervention packages [2]. Whilst many studies focus on the vector (resistance, time and place of biting), the critical component of how human behaviour impacts the efficacy of vector control tools has been largely overlooked for IRS. In this article, after illustrating the importance of IRS in malaria control and discussing the current indicators that are used to evaluate its impact, the focus will 
shift to a critical indicator that is currently not measured: Changes made to insecticide-treated walls (wall modifications) by household owners over time following IRS implementation.

IRS and the fight against malaria: past, present, and future Indoor residual spraying (IRS) has been a key intervention in the fight against malaria mosquitoes since the discovery in 1939 that chemicals like DDT had insecticidal properties and could aid public health by reducing the vector population size [9]. To reduce the high malaria burden in the 1960s, the World Health Organization (WHO) introduced large scale use of IRS to target indoor resting mosquitoes as the primary vector control method [9]. To supplement this effort, methods such as larval control and building mosquito-proofed houses [10] were encouraged too. However, IRS was later reduced to fewer countries due to challenges such as insecticide resistance due to over-reliance on one class of insecticide (the organochloride DDT), logistical constraints, sustainability, economic and financial issues [10].

However, in 2006, the WHO recommended IRS again as a tool to reduce or interrupt local malaria transmission in different epidemiological settings. As a result, several countries introduced and/or expanded their IRS programmes [11], with dedicated funding from President's Malaria Initiative (PMI) and the Global Fund to Fight AIDS, Tuberculosis and Malaria (GFATM). IRS is believed to have contributed to about $14 \%$ and $16 \%$ of overall malaria cases prevented and reduction in malaria prevalence, respectively, between 2000 and 2015 in the WHO Africa region [1]. As IRS remains an integral part of vector control efforts, partly because of the widespread resistance to pyrethroids used in LLINs [12, 13], huge investments are channeled to it $[3,14]$.

\section{How do we currently monitor IRS efficacy?}

The effectiveness of LLINs and IRS relies on (1) the biology and behaviour of mosquito vectors (their biting and resting behaviour as well as their susceptibility status to insecticides), (2) the insecticide(s) selected (active ingredient, bio-efficacy (in case of LLINs) and residual efficacy (IRS) over time), durability (LLINs), and (3) the acceptability and use of the interventions as well as their coverage [15-18]. The latter, coverage, is normally estimated by calculating the proportion of the households that own 1 LLIN per 2 persons, or the proportion of houses or structures or households that have been sprayed in the case of IRS [19-22]. A certain coverage threshold (believed to be $60 \%$ for LLINs [17] and $80 \%$ for IRS [19, 22 ] is envisioned for the tools to work optimally as they work at a community level: the greater the coverage, the higher the impact $[18,22,23]$.

For LLINs, the most widely used vector control intervention, there are clear guidelines on how to monitor relevant indicators to assess their protective efficacy postdistribution [21, 22, 24]. These indicators, which include coverage, usage, net maintenance and killing efficacy, are routinely monitored at an annual interval after net distribution [25-27]. However, for IRS, apart from assessing the initial coverage (at time of application), only the quality of the IRS product may be assessed post-spraying through standard residual efficacy tests using WHO cone bioassays $[19,28]$. This is, however, not standard practice in all malaria-endemic countries, and if implemented, often only carried out in a handful of houses per province or even country, and halted when mosquito mortality falls below $80 \%$.

LLIN studies show that community acceptance and uptake is key to the success of vector control. For IRS, advocacy campaigns before the implementation of IRS are geared towards preparing communities for IRS to achieve high initial coverage. However, after a house/ structure has been sprayed, there is no follow-up on whether the treated surfaces remain covered until the next spray cycle (or at least up to the point where the insecticide lost its residual efficacy). The WHO and other institutions have not yet set forth guidelines to aid national malaria control programmes (NMCPs) and partners on how to monitor post-spray owner compliance. Current guidelines to assess the effectiveness of IRS programmes include initial coverage (number of structures covered) and the residual efficacy of the product sprayed [19] (Table 1). For simplicity, here it is assumed that adequate entomological surveillance is in place, to ensure that targeted vector populations do rest indoors, and are susceptible to the insecticides selected (although this is unlikely always the case).

\section{IRS and society: the human attitude and behaviour towards IRS}

One of the WHO-recommended, but not required, indicators for effective implementation of IRS is 'social performance'. Social studies that exist assess the acceptability of IRS prior and/or during the implementation phase, to understand why IRS is refused and to evaluate if action can be taken to mitigate this (i.e. through improved community awareness) [29-32]. As alluded to before, LLIN indicators such as usage, coverage, and durability are monitored annually post-distribution using existing guidelines [20,21]. But when it comes to IRS, once spraying is done, only monitoring of the product's residual efficacy occurs sporadically via wall bio-assays. The extent to which insecticide-treated wall 
Table 1 Currently recommended indicators for monitoring the effectiveness of IRS programmes

\begin{tabular}{|c|c|c|}
\hline Program & IRS indicator & Reference \\
\hline World Health Organization (WHO) & $\begin{array}{l}\text { Coverage: 'the proportion of structures/houses sprayed } \\
\text { in relation to those not sprayed (proportion of struc- } \\
\text { tures/houses sprayed in relation to those targeted for } \\
\text { spraying)'. This is done at the district, region, country, } \\
\text { province, and global scale } \\
\text { Residual efficacy: 'the quality of IRS, impact, insecticide } \\
\text { dosage and longevity on treated surfaces is routinely } \\
\text { measured by WHO cone bioassay using susceptible } \\
\text { strains maintained at a central laboratory' } \\
\text { Social performance: 'the perception of the community } \\
\text { towards IRS can be assessed through community } \\
\text { knowledge, attitude, behavior and practice (KABP) } \\
\text { surveys'. Such surveys are not required to run an } \\
\text { IRS program but are reserved for situations where } \\
\text { community-related IRS problem may arise }\end{array}$ & {$[48]$} \\
\hline Roll Back Malaria (RBM) & $\begin{array}{l}\text { Population coverage: 'Proportion of households sprayed } \\
\text { by IRS in the last } 12 \text { months' }\end{array}$ & {$[49]$} \\
\hline UNICEF & $\begin{array}{l}\text { Households covered by vector control: 'Proportion of } \\
\text { households sprayed by IRS in the last } 12 \text { months'. } \\
\text { Universal coverage of vector control:'Proportion of } \\
\text { households sprayed by IRS within the last } 12 \text { months' }\end{array}$ & {$[50]$} \\
\hline
\end{tabular}

surfaces are modified post-application, and why, are currently not being monitored, yet home-owners are known to wash and/or brush, (re)plaster and/or (re) paint their walls over time. These activities occur for a variety of reasons, such as the dislike of the smell or coloration of walls after IRS application, mistrusting the government/implementers [33, 34] or due to non-IRS related factors such as scheduled maintenance/renovations and religious/cultural practices [35] (Fig. 1). As this particular indicator (coverage over time post-spraying) is not routinely monitored by NMCPs programmes and/or partners, it is impossible to understand its significance and its impact.

However, two previous studies have shown that IRS coverage can decrease rapidly over time as a result of wall modifications post-spraying. Figure 2 shows data from South Africa (IRS with deltamethrin in 1999) and India (IRS with DDT in 2004). In both studies, IRS coverage (here defined as walls left untouched after IRS application) rapidly declined post-application. In South Africa, coverage decreased to $50 \%$ within 3 months after spraying [36] (Fig. 2a), and in India, 80\% of the houses were replastered within two to 3 months following the IRS

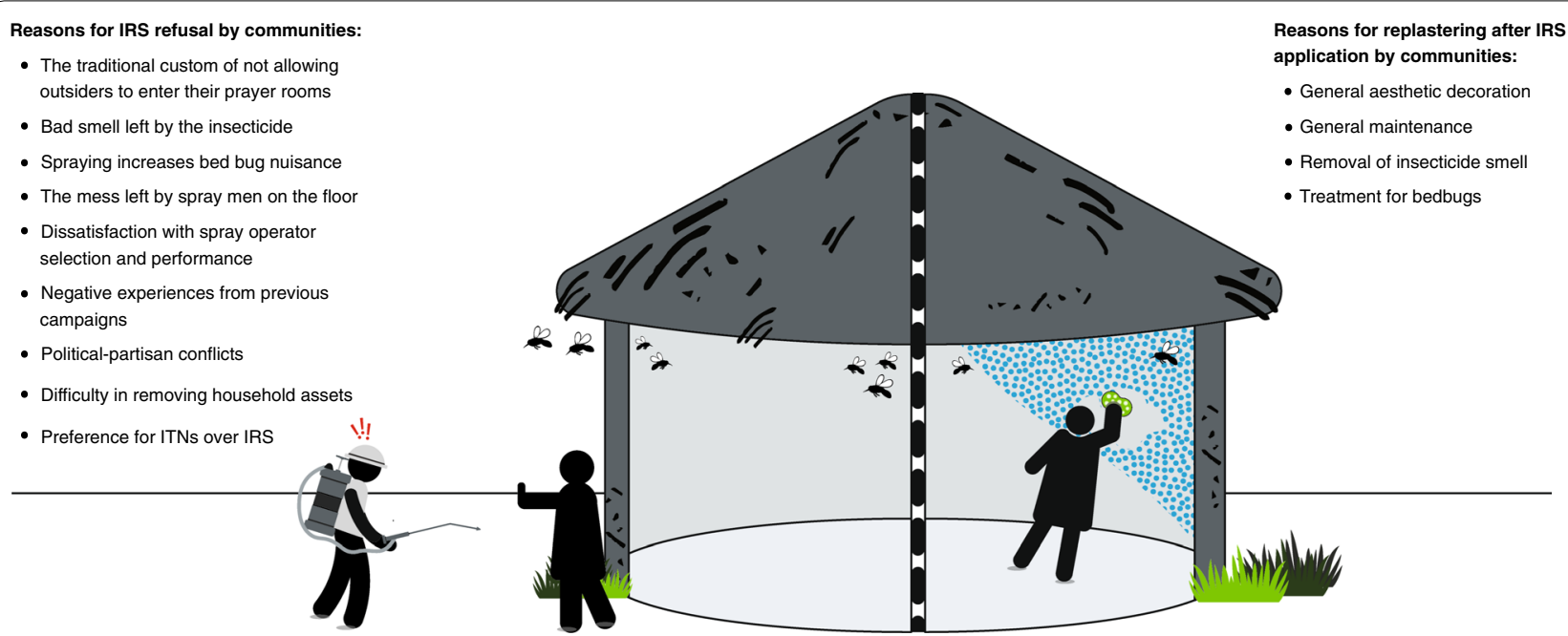

Fig. 1 Some reasons for IRS refusal (on the left) and for wall modifications post-application (on the right) by communities [32, 33] 

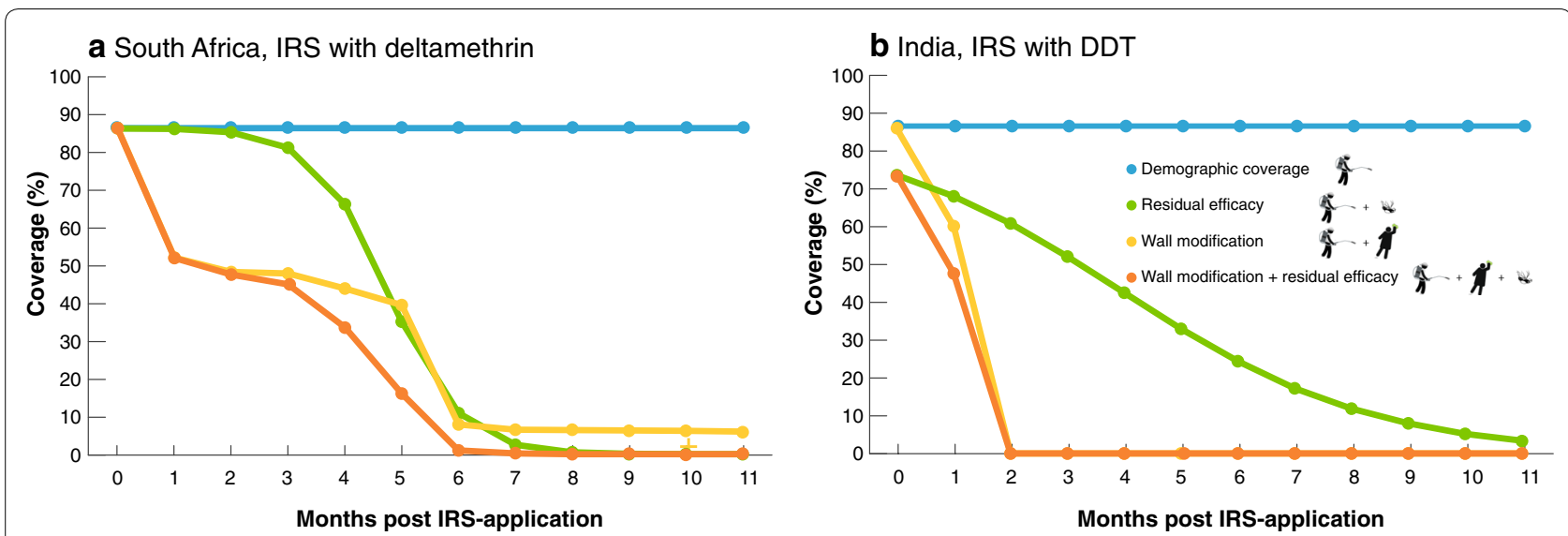

Fig. 2 The effect of wall modifications and waning residual efficacy on the actual coverage of IRS campaigns. a Data from mud houses in South Africa (IRS with deltamethrin in 1999) and b from Koraput district in India (IRS with DDT 2004). The blue line represents actual (demographic or initial coverage as reported by programs and/or partners determined at the time of spraying), the yellow line represents observed wall modifications following real IRS-applications, the green line represents the residual bioefficacy on non-modified walls as monitored through WHO cone bioassays and the orange line represents the predicted impact of wall modifications combined with a waning residual efficacy on the effective IRS coverage. Logistic binomial models were fitted through actual residual bioefficacy data, obtained from (deltamethrin) [15] and (DDT) [43]. Bayesian models were fitted using Hamiltonian Monte Carlo sampling methods [51, 52]. Four chains were initialised to assess the convergence of 1000 iterations, the first 500 of each were discarded as burn in. The posterior distributions of parameters (4000 iterations) and 90\% Bayesian credible intervals were estimated, posterior checks were performed using shinystan (version 1.0.0) [53] and visually confirmed to fit the data. The caveats for the South Africa data are: (1) repeated measures, as walls were washed or replastered on more than one occasion during the year, which leads to an overrepresentation of the extent of wall modifications, (2) initial coverage not being reported, necessitating us to use the self-reported $86.6 \%$ for IRS during the past 2 years, (3) no information on start date of IRS, so we assumed month 1 to be November, matching text with table. The caveat for the data from India includes initial coverage (reported for households and structures, we opted to show household-level data, as modification data were also reported at that level). For both studies it is not clear (probably not assessed) if all rooms and all walls in each room had been modified, or if only part of the house/structure was affected. Note that additional data (South Africa, concrete houses; India, Malkangiri district) are shown in Additional file 1: Fig. S1, and show similar patterns

campaign (Fig. 2b) [33]. By month 3 or 4 (depending on the district) walls in all the sprayed houses had been replastered [33] (Fig. 2b). Although there may be some caveats with the study designs (see the legend of Fig. 2), the figures clearly illustrate there may be a serious challenge with IRS if communities today behave in similar ways.

\section{What is the true impact of the IRS?}

To date, the studies from India and South Africa provide the only longitudinal data on wall modifications post-IRS. Whilst it is not known how representative the data from India and South Africa are to other settings in Africa where IRS has been or is being implemented, wall modifications could reduce or potentially completely remove the bioavailability of insecticides. This may change the outcomes of IRS impact evaluations, as this indicator is not included in models that estimate the efficacy (i.e. number of structures/houses sprayed and/or the number of people protected) of IRS campaigns [14, 37-39]. These estimates are mainly based on initial coverage, the vector susceptibility status to insecticides, and the residual efficacy of the product used [23, 38, 40]. In addition, studies that aim to evaluate the additional benefits of
IRS combined with LLINs have concluded that no or little incremental benefits exist when the two interventions are combined [41, 42]. These latter studies have, however, only considered initial coverage and could have underestimated the additional protective value of two interventions combined, if study communities modified their walls post-IRS application.

Apart from changes in coverage post-spraying, there is little known about the impact of wall modifications on the residual efficacy of the insecticide. This will likely depend on a variety of factors, including the type and extent of the modification, as well as the materials used to prepare and modify the walls. Replastered walls have been shown to still kill malaria vectors, although mortality was significantly lower on replastered walls [33]. Having said that, looking at the patterns in Fig. 2, and considering the residual efficacy of deltamethrin (which ranges from 1 to 3 months) [15] and DDT (2 months) [43] it is fair to assume that the observed wall modification behaviours in South Africa and India will have impacted control efforts. To illustrate this, existing data on wall modifications and residual efficacy are combined in a single plot (Fig. 2), assuming that waning residual efficacy equals loss in coverage (i.e. 
$70 \%$ mortality in cone bio-assays translates to $70 \%$ of the houses killing all mosquitoes, while $30 \%$ kill none). The situation is more complex, as different houses have different residual efficacies and attract different numbers of different vector species, but Fig. 2 illustrates how wall modification could severely impact IRS efficacy, and hence the actual number of people protected.

Although deltamethrin (and other pyrethroids) and DDT are now less frequently used in IRS campaigns due to resistance and/or health concerns [12, 28, 44-46] Actellic ${ }^{\circledR} 300 \mathrm{CS}$ (Syngenta, Switzerland), SumiShield ${ }^{\circledR} 50$ WG (Sumitomo, Japan) [14, 47], both with residual efficacies of $>6$ months, will not be as impactful as currently assumed if household members modify their walls post-spraying.

\section{Conclusions}

To understand the true impact of IRS campaigns, IRS coverage post-implementation needs to be evaluated as part of Monitoring and Evaluation activities, to estimate the number of people protected. By creating guidelines for malaria control programmes and partners, the extent of wall modifications following a successful IRS campaign can be monitored and appropriate actions taken to either prevent or mitigate unwanted human behaviours. These include actions like improved community engagement and education, and/or re-spraying of modified walls. This will ensure that IRS campaigns achieve optimal protection, thereby reducing malaria morbidity and mortality even further.

\section{Supplementary information}

Supplementary information accompanies this paper at https://doi. org/10.1186/s12936-020-3102-6.

Additional file 1: Figure S1. The effect of wall modifications and waning residual efficacy on the actual coverage of IRS campaigns.

\section{Abbreviations \\ LLINs: long-lasting insecticidal nets; ITNs: insecticide-treated nets; IRS: indoor residual spraying; GTS: Global Technical Strategy; DDT: Dichlorodiphenyl- trichloroethane; WHO: World Health Organization; GMEP: Global Malaria Eradi- cation Program; PMI: President Malaria Initiative; NgenIRS: next-generation indoor residual spraying; GFATM: Global Fund to Fight AIDS, Tuberculosis and Malaria; PMI AIRS: President Malaria Initiative Africa Indoor Residual Spraying; RBM: Roll Back Malaria; UNICEF: United Nations International Children's Emer- gency Fund; CISM: Centro de Investigação de Saúde de Manhiça.}

\section{Acknowledgements}

We notable thank Dr. Ellie Sherrard-Smith (Medical Research Centre for Global Infectious Disease Analysis, Department of Infectious Disease Epidemiology, Faculty of Medicine, Imperial College, London, UK) for her support with creating Fig. 2 and Lucía Fernández Montoya (Barcelona Institute for Global Health, Spain) for commenting on the manuscript. Illustrations (Figs. 1 and 2) were created by Megan Joyce (Vislab, School of Life Sciences, Arizona State University, USA).

\section{Authors' contributions}

Conception of the idea and literature search: $\mathrm{MO}, \mathrm{KP}$; writing the first draft $\mathrm{MO}$; review and final draft: MO, KP. All authors read and approved the final manuscript.

\section{Funding}

MO and KP were supported by the Bill and Melinda Gates Foundation and Obra Social "la Caixa" Partnership for the Elimination of Malaria in Southern Mozambique (OPP1115265). ISGlobal is a member of the CERCA Programme, Generalitat de Catalunya (http://cerca.cat/en/suma/). CISM is supported by the Government of Mozambique and the Spanish Agency for International Development (AECID).

\section{Availability of data and materials}

Data shown in this article (Fig. 2) are obtained from the scientific literature and all citations can be found in the bibliography.

\section{Ethics approval and consent to participate}

Not applicable.

\section{Consent for publication}

Not applicable.

\section{Competing interests}

The authors declare that they have no competing interests.

\section{Author details}

${ }^{1}$ ISGlobal, Hospital Clinic, University of Barcelona, Barcelona, Spain. ${ }^{2}$ Centro de Investigação em Saúde de Manhiça (CISM), Maputo, Mozambique. ${ }^{3}$ Center for Evolution and Medicine, School of Life Sciences, Arizona State University, Tempe, USA. ${ }^{4}$ The Biodesign Center for Immunotherapy, Vaccines, and Virotherapy, Arizona State University, Tempe, AZ, USA.

Received: 1 October 2019 Accepted: 7 January 2020

Published online: 17 January 2020

\section{References}

1. Bhatt S, Weiss DJ, Cameron E, Bisanzio D, Mappin B, Dalrymple U, et al. The effect of malaria control on Plasmodium falciparum in Africa between 2000 and 2015. Nature. 2015;526:207-11.

2. Dhiman S. Are malaria elimination efforts on the right track? An analysis of gains achieved and challenges ahead. Infect Dis Poverty. 2019;8:14.

3. WHO. World Malaria Report 2018. Geneva: World Health Organization; 2018. https://www.who.int/malaria/publications/world-malaria-repor t-2018/report/en/. Accessed 17 July 2019.

4. WHO. Global technical strategy for malaria elimination. Geneva: World Health Organization; 2015. https://www.who.int/malaria/publications/ atoz/9789241564991/en/. Accessed 15 June 2019.

5. Msellemu D, Shemdoe A, Makungu C, Mlacha Y, Kannady K, Dongus S, et al. The underlying reasons for very high levels of bed net use, and higher malaria infection prevalence among bed net users than non-users in the Tanzanian city of Dar es Salaam: a qualitative study. Malar J. 2017;16:423.

6. Ranson H, Abdallah H, Badolo A, Guelbeogo WM, Kerah-Hinzoumbé C, et al. Insecticide resistance in Anopheles gambiae: data from the first year of a multi-country study highlight the extent of the problem. Malar J. 2009:8:299.

7. Killeen GF, Ranson H. Insecticide-resistant malaria vectors must be tackled. Lancet. 2018;21(391):1551-2.

8. Killeen GF. Characterizing, controlling and eliminating residual malaria transmission. Malar J. 2014;13:330.

9. Sadasivaiah S, Tozan Y, Breman JG. Dichlorodiphenyltrichloroethane (DDT) for indoor residual spraying in Africa: how can it be used for malaria control. Am J Trop Med Hyg. 2007;77(6):249-63.

10. Mabaso ML, Sharp B, Lengeler C. Historical review of malarial control in southern African with emphasis on the use of indoor residual housespraying. Trop Med Int Health. 2004;9:846-56.

11. Oxborough RM. Trends in US President's Malaria initiative-funded indoor residual spray coverage and insecticide choice in sub-Saharan Africa (2008-2015): urgent need for affordable, long-lasting insecticides. Malar J. 2016;15:146. 
12. Hemingway J, Ranson H, Magill A, Kolaczinski J, Fornadel C, Gimnig J, et al. Averting a malaria disaster: will insecticide resistance derail malaria control? Lancet. 2016;387:1785-8.

13. Sherrard-Smith E, Griffin JT, Winskill P, Corbel V, Pennetier C, Djénontin A, et al. A systematic review of indoor residual spray efficacy and effectiveness against Plasmodium falciparum in Africa. Nat Commun. 2018;26(9):4982.

14. PMI. Presidents malaria initiative. 2018. https://www.pmi.gov/resource-libra ry/partner-reports. Accessed 17 July 2019.

15. Dengela D, Seyoum A, Lucas B, Johns B, George K, Belemvire A, et al. Multicountry assessment of residual bio-efficacy of insecticides used for indoor residual spraying in malaria control on different surface types: results from program monitoring in 17 PMI/USAID-supported IRS countries. Parasit Vectors. 2018;11:71.

16. WHO. World malaria report 2017. Geneva: World Health Organization; 2017. https://www.who.int/malaria/publications/world-malaria-report-2017/en/. Accessed 17 July 2019

17. Killeen GF, Marshall JM, Kiware SS, South AB, Tusting LS, Chaki PP, et al. Measuring, manipulating and exploiting behaviors of adult mosquitoes to optimize malaria vector control impact. BMJ Global Health. 2017;2:e000212.

18. Killeen GF, Seyoum A, Gimnig JE, Stevenson JC, Drakeley CJ, Chitnis N. Made-to-measure malaria vector control strategies: rational design based on insecticide properties and coverage of blood resources for mosquitoes. Malar J. 2014;13:146.

19. WHO. An operational manual for indoor residual spraying (IRS) for malaria transmission and elimination. Geneva, Switzerland: World Health Organization; 2015.

20. WHO. Guidelines for monitoring the durability of long-lasting insecticidal mosquito nets under operational conditions. 2011. https://www.who.int/ malaria/publications/atoz/9789241550499/en/. Accessed 15 May 2019.

21. WHO. Methods for maintaining coverage with long-lasting insecticidal nets (LLINs). 2013. https://www.who.int/malaria/mpac/mpac_sp13_vcteg_unive rsal_llin_coverage_report.pdf?ua=1. Accessed 15 May 2019.

22. WHO. Guidelines for malaria vector control. 2019. https://www.who.int/ malaria/publications/atoz/9789241550499/en/. Accessed 10 May 2019.

23. Larsen DA, Borrill L, Patel R, Fregosi L. Reported community-level indoor residual spray coverage from two-stage cluster surveys in sub-Saharan Africa. Malar J. 2017;16:249.

24. Tokponnon FT, Aholoukpe B, Denon EY, Gnanguenon V, Bokossa A, N'Guessan R, et al. Evaluation of the coverage and effective use rate of longlasting insecticidal nets after nation-wide scale-up of their distribution in Benin. Parasit Vectors. 2013;6:265.

25. Plucinski MM, Chicuecue S, Macete E, Colborn J, Yoon SS, Kachur SP, et al. Evaluation of a universal coverage bed net distribution campaign in four districts in Sofala Province, Mozambique. Malar J. 2014;13:427.

26. Koenker H, Arnold F, Ba F, Cisse M, Diouf L, Eckert E, et al. Assessing whether universal coverage with insecticide-treated nets has been achieved: is the right indicator being used? Malar J. 2018;17:355.

27. Liu H, Xu JW, Guo XR, Havumaki J, Lin YX, Yu GC, et al. Coverage, use and maintenance of bed nets and related influence factors in Kachin Special Region II, northeastern Myanmar. Malar J. 2015;14:212.

28. WHO. Specifications and evaluations for public health pesticides. Geneva: World Health Organization; 2006. http://www.who.int/whopes/quality/ newspecif/en/. Accessed 17 July 2019.

29. Munguambe K, Pool R, Montgomery C, Bavo C, Nhacolo A, Fiosse L, et al. What drives community adherence to indoor residual spraying (IRS) against malaria in Manhiça district, rural Mozambique: a qualitative study. Malar J. 2011:10:344.

30. Ingabire CM, Rulisa A, Van Kempen L, Muvunyi C, Koenraadt CJM, Van Vugt $M$, et al. Factors impeding the acceptability and use of malaria preventive measures: implications for malaria elimination in eastern Rwanda. Malar J. 2015;14:136.

31. Manana PN, Kuonza L, Musekiwa A, Mpangane HD, Koekemoer LL. Knowledge, attitudes and practices on malaria transmission in Mamfene, KwaZulu-Natal Province, South Africa 2015. BMC Public Health. 2017;18:41.

32. Magaço A, Botão C, Nhassengo P, Saide M, Ubisse A, Chicumbe S, et al. Community knowledge and acceptance of indoor residual spraying for malaria prevention in Mozambique: a qualitative study. Malar J. 2019;18:27.

33. Gunasekaran K, Sahu S, Jambulingam P, Das P. DDT indoor residual spray, still an effective tool to control Anopheles fluviatilis-transmitted Plasmodium falciparum malaria in India. Trop Med Int Health. 2005;10:160-8.
34. Hlongwana KW, Mavundza EJ, Mohapi EP, Kruger P, Urbach J, Mukaratirwa $\mathrm{S}$, et al. Vector-control personnel's knowledge, perceptions and practices towards insecticides used for indoor residual spraying in Limpopo Province, South Africa. Parasit Vectors. 2013;6:118.

35. Hlongwana KW, Zitha A, Mabuza AM, Maharaj R. Knowledge and practices towards malaria amongst residents of Bushbuckridge, Mpumalanga, South Africa. Afr J Primary Health Care Fam Med. 2011;3:257.

36. Govere J, Durrheim D, la Grange K, Mabuza A, Booman M. Community knowledge and perceptions about malaria and practices influencing malaria control in Mpumalanga Province, South Africa. S Afr Med J. 2000;90:611-6.

37. Yakob L, Dunning R, Yan G. Indoor residual spray and insecticide-treated bednets for malaria control: theoretical synergisms and antagonisms. J R Soc Interface. 2010;8:799-806.

38. Kiware SS, Chitnis N, Tatarsky A, Wu S, Castellanos HMS, Gosling R, et al. Attacking the mosquito on multiple fronts: insights from the vector control optimization model (VCOM) for malaria elimination. PLOS ONE. 2017;12:e0187680

39. Griffin JT, Hollingsworth TD, Okell LC, Churcher TS, White M, Hinsley W, et al. Reducing Plasmodium falciparum malaria transmission in Africa: a modelbased evaluation of intervention strategies. PLoS Med. 2010;7:e1000324.

40. Kiware SS, Chitnis N, Devine GJ, Moore SJ, Majambere S, et al. Biologically meaningful coverage indicators for eliminating malaria transmission. Biol Lett. 2012;8:874-7.

41. Okumu FO, Kiware SS, Moore SJ, Killeen GF. Mathematical evaluation of the community-level impact of combining bed nets and indoor residual spraying upon malaria transmission in areas where the main vectors are Anopheles arabiensis mosquitoes. Parasit Vectors. 2013;6:17.

42. Okumu FO, Moore SJ. Combining indoor residual spraying and insecticidetreated nets for malaria control in Africa: a review of possible outcomes and an outline of suggestions for the future. Malar J. 2011;10:208.

43. Okumu FO, Chipwaza B, Madumla EP, Mbeyela E, Lingamba G, Moore J, et al. Implications of bio-efficacy and persistence of insecticides when indoor residual spraying and long-lasting insecticide nets are combined for malaria prevention. Malar J. 2012;11:378.

44. Ranson H, Lissenden N. Insecticide resistance in African Anopheles mosquitoes: a worsening situation that needs urgent action to maintain malaria control. Trends Parasitol. 2016;32:187-96.

45. Ranson H, N'Guessan R, Lines J, Moiroux N, Nkuni Z, Corbel V. Pyrethroid resistance in African anopheline mosquitoes: what are the implications for malaria control? Trends Parasitol. 2011;27:91-8.

46. Hertz-Picciotto I, Sass JB, Engel S, Bennett DH, Bradman A, Eskenazi B, et al. Organophosphate exposures during pregnancy and child neurodevelopment: recommendations for essential policy reforms. PLoS Med. 2018;15:e1002671.

47. Chinula D, Hamainza B, Chizema E, Kavishe DR, Sikaala CH, Killeen GF. Proportional decline of Anopheles quadriannulatus and increased contribution of An. arabiensis to the An. gambiae complex following introduction of indoor residual spraying with pirimiphos-methyl: an observational, retrospective secondary analysis of pre-existing data from south-east Zambia. Parasit Vectors. 2018;11:544.

48. WHO. An operational manual for indoor residual spraying (IRS) for malaria transmission and elimination. Geneva, Switzerland: World Health Organization; 2013.

49. RBM. Guidelines for core population-based indicators. 2009. https://relie fweb.int/sites/reliefweb.int/files/resources/AC719D00E5DE6F5D492575B30 01BE5A3-RMB-guideline-20009.pdf. Accessed 17 July 2019.

50. UNICEF. Household survey indicator for malaria control. 2013. http://data. unicef.org/wp-content/uploads/2015/12/HouseholdSurveylndicatorsForMa lariaControl_179.pdf. Accessed 17 July 2019.

51. Papaspiliopoulos $O$, Roberts GO, Sköld M. A general framework for the parametrization of hierarchical models. Stat Sci. 2007;22:59-73.

52. Betancourt M, Girolami M. Hamiltonian Monte Carlo for hierarchical models. In: Upadhyay SK, Singh U, Dey DK, Logananthan A, editors. Current trends in Bayesian methodology with applications. New York: Chapman and Hall CRC Press; 2015.

53. Team SDS. Modeling language. Users guide. Ref Man. 2015:1-488 det.

\section{Publisher's Note}

Springer Nature remains neutral with regard to jurisdictional claims in published maps and institutional affiliations. 